\title{
Profile of sapacitabine: potential for the treatment of newly diagnosed acute myeloid leukemia in elderly patients
}

This article was published in the following Dove Press journal:

Clinical Interventions in Aging

6 May 2014

Number of times this article has been viewed

\section{Ming Y Lim \\ Katarzyna Jamieson}

Department of Medicine, Division of Hematology/Oncology, University of North Carolina, Chapel Hill, NC, USA
Correspondence: Katarzyna Jamieson University of North Carolina School of Medicine, Physicians Office Building, CB\# 7305, 170 Manning Drive,

3rd Floor, Chapel Hill, NC, USA

Tel +l 3523289355

Email katarzyna_jamieson@med.unc.edu
Abstract: Acute myeloid leukemia (AML) is a hematopoietic stem cell disorder that affects approximately 14,000 persons each year in the US. AML occurs at all ages but the incidence increases with age with the median age at diagnosis being 67 years. Advances in the treatment of AML over the past decades have led to improved survival, albeit mostly in younger patients. The prognosis of older patients with this disease over the same time span has not changed much and remains dismal. This review focuses on the epidemiology and characteristics of AML in elderly patients, the rationale for treating elderly AML patients, and the currently available and potential future treatment options such as sapacitabine. Elderly AML patients treated with intensive chemotherapy have a higher mortality rate, and a lower rate of complete remission and overall survival when compared to the younger population. This is due to both the different biology of the disease and the number of patient-specific factors. However, elderly AML patients treated with aggressive chemotherapy can achieve durable remissions, which offer prolonged survival and improved quality of life. Recent data also indicates that elderly AML patients deemed unfit for intensive chemotherapy benefit from leukemia-specific attenuated dose chemotherapy compared to supportive care alone. This has led to renewed interest to look for anti-leukemic therapies designed specifically for older patients. Sapacitabine, a novel oral nucleoside analog, promises good efficacy, favorable toxicity profile, and ease of administration; all of which makes it very appealing. Results from pre-clinical and clinical studies have been very encouraging and sapacitabine is currently being evaluated in a Phase III study, of which the results are eagerly awaited.

Keywords: AML, elderly, management, sapacitabine

\section{Introduction}

Acute myeloid leukemia (AML) is a hematopoietic stem cell disorder characterized by clonal proliferation of myeloid precursors with an inhibition in differentiation, leading to accumulation of immature cells at various stages and reduction in the production of normal hematopoietic components: erythrocytes, platelets, and mature granulocytes. With an incidence of approximately four per 100,000 per year, AML is relatively uncommon but responsible for a large number of cancer-related deaths. ${ }^{1}$ It is estimated that in the US, about 14,590 persons will be diagnosed with and 10,370 will die of AML in 2013. The incidence of AML increases with age; the median age at diagnosis today is 67 years (Figure 1), making AML truly "a disease of the elderly."

The past 4 decades have seen significant advances in the diagnosis and treatment of this condition. The succession of clinical trials performed throughout previous decades established the standard of care for the treatment of this disease: at present, 


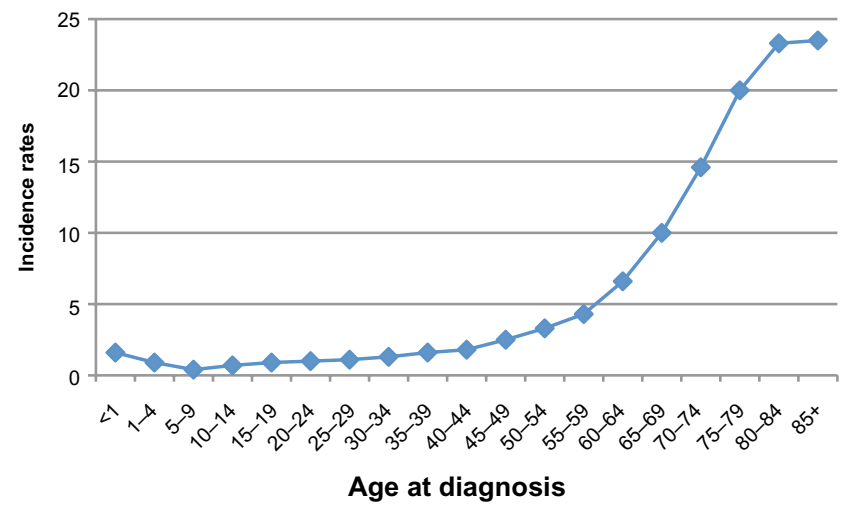

Figure I Age-specific SEER incidence rates for acute myeloid leukemia, 2006-20I0. Abbreviation: SEER, Surveillance, Epidemiology and End Results.

as many as $75 \%$ of patients with newly diagnosed AML can expect to enter remission and as many as 40\%-50\% can expect to survive long-term. However, the great majority of patients enrolled in those trials were younger than 60 years old, and as expected, the chemotherapy regimens developed in the process perform best in the younger patient population. Very few older patients can tolerate such intensive chemotherapy; those who can, do not respond as well as younger patients, relapse sooner, and their long-term prognosis remains poor.

Over the past several years, we have come to a realization that improving the outcomes of older patients with AML will require approaches specifically developed for them. These approaches will need to take into account not only the different biology of AML in these patients but also find a way to overcome specific host factors such as comorbid conditions, overall fitness, and difficult psychosocial aspects. In this review, we will provide an overview on the epidemiology and characteristics of AML in elderly patients, the rationale for treating elderly AML patients, and the potential of sapacitabine to become a novel treatment strategy, specifically for elderly patients.

\section{Epidemiology and treatment outcome in elderly AML patients}

Prior to the introduction of effective therapy, AML was a uniformly fatal disease, with an expected 5-year survival of less than $10 \%$. Over the past 3 decades, the survival rate in AML patients gradually increased: according to the Surveillance, Epidemiology and End Results (SEER) program, statistics between 2003 and 2009 reached 24.9\% (Figure 2). ${ }^{1}$ The 5-year relative survival of younger AML patients actually increased to $41.6 \%$. At the same time, the 5-year survival of older patients aged $\geq 65$ years remained poor at $5.4 \%$ (Figure 3 ). ${ }^{1}$ Other registries and AML treatment groups confirmed the slow but

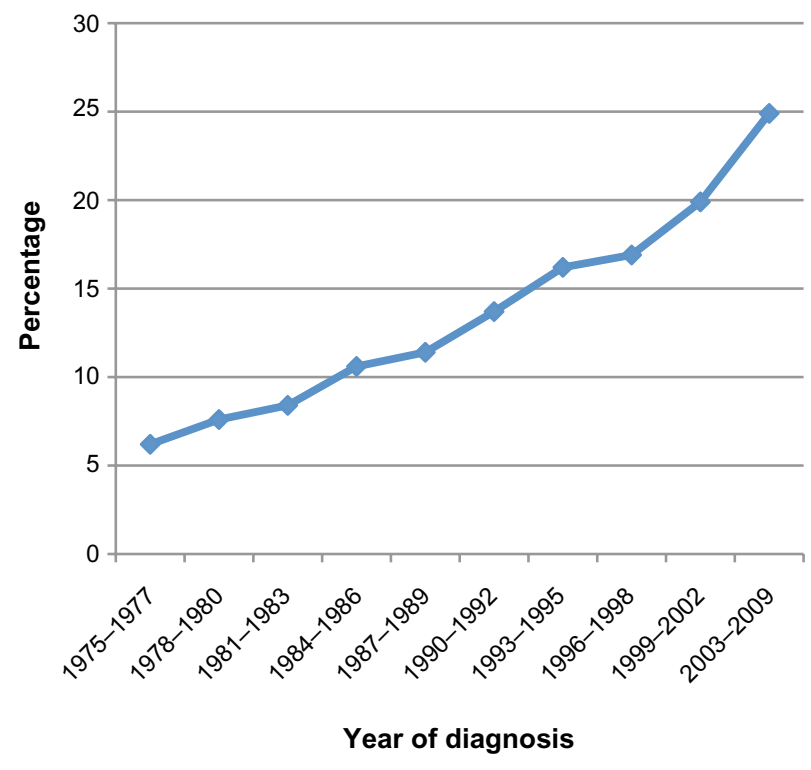

Figure 2 Five-year relative survival (percentage) for acute myeloid leukemia by year of diagnosis.

steady improvement over time in the outcomes of younger patients coupled with the lack of any appreciable progress in older patients (Figure 4). ${ }^{2,3}$ The newer data also confirmed the arbitrary nature of the cut-off we use to define younger and older patient populations, which is between 55 and 65 years old. It has become apparent that beginning at the age of 30-40 years old, the increasing age of these patients acts as an independent continued variable until well past age 65 years. The retrospective analysis of 968 untreated AML adults from the Southwest Oncology Group trials demonstrated a consistent decrease with age in the likelihood of achieving complete remission (CR) and the likelihood of overall survival (OS). Among patients $<56,56-65,66-75$, and $>75$ years the CR and OS rates were respectively: $64 \%, 46 \%, 39 \%$, and $33 \%$ and 18.8, 9.0, 6.9, and 3.5 months. ${ }^{4}$ These numbers are particularly sobering considering that they reflect the outcomes of a selected minority $(<30 \%)$ of older patients with AML who

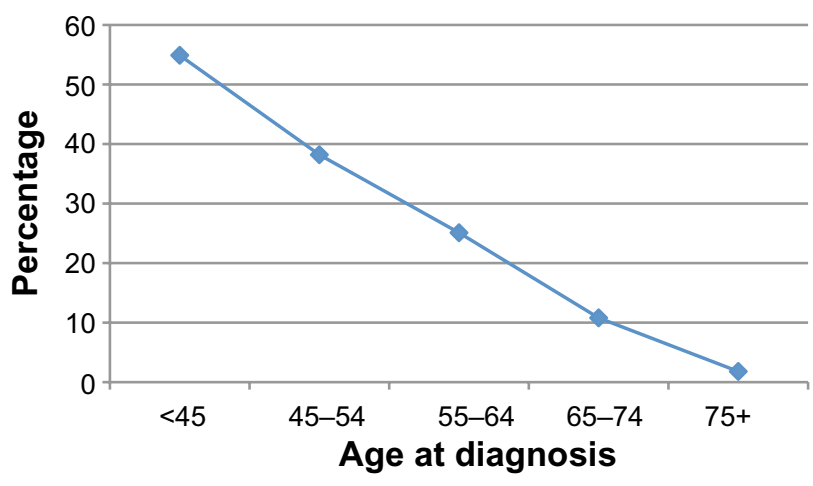

Figure 3 Five-year relative survival (percentage) by age of diagnosis, 2003-2009. 
Improvements in survival over time 1970 to 2005
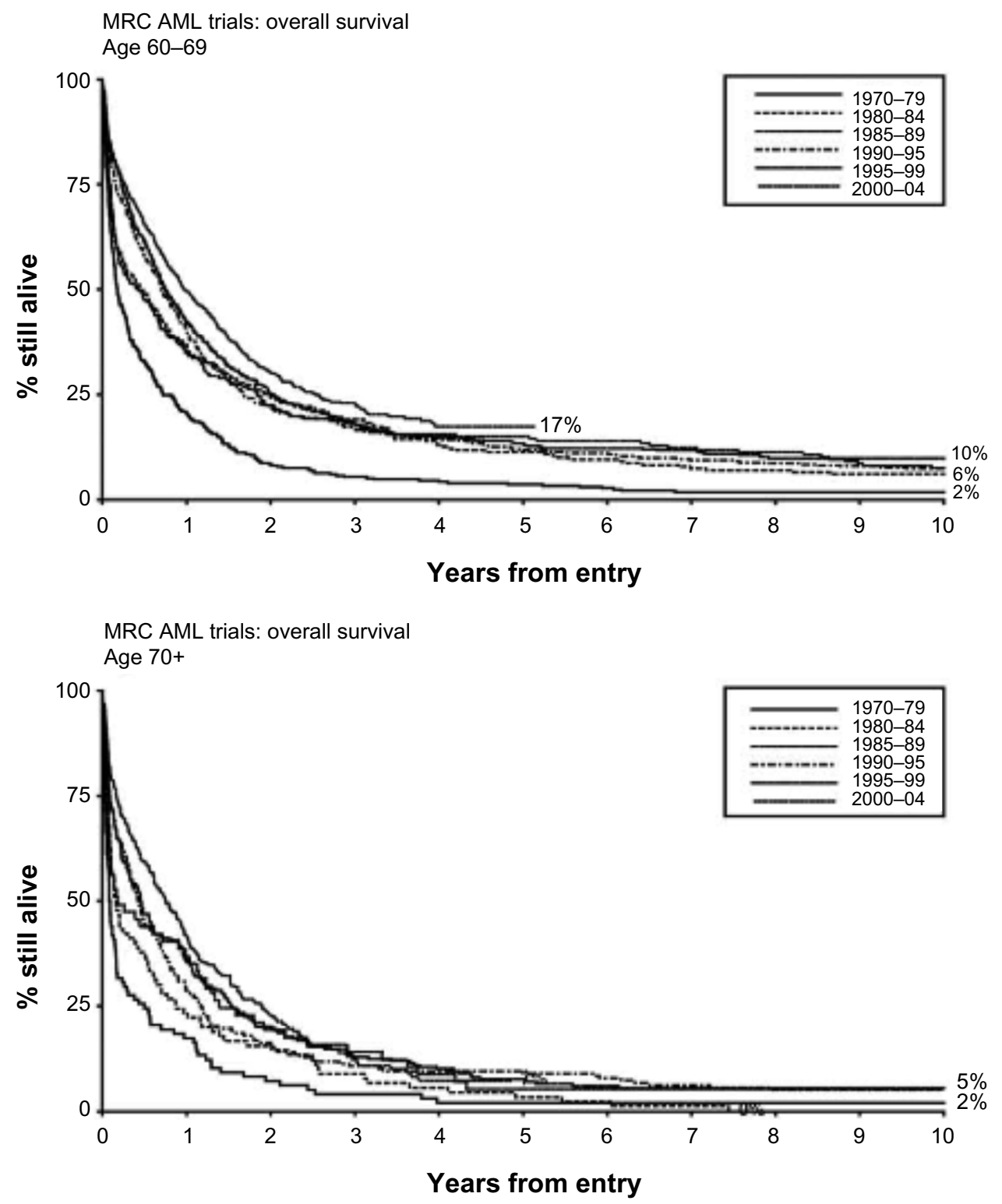

Figure 4 Changes in outcome in older patients over 3 decades.

Note: Reprinted from Semin Hematol, 43(2), Burnett AK, Mohite U, Treatment of older patients with acute myeloid leukemia - new agents, 96-106. ${ }^{2}$ Copyright $\odot$ 2006, with permission from Elsevier.

Abbreviations: AML, acute myeloid leukemia; MRC, Medical Research Council.

were deemed "fit" to receive intensive therapy, and therefore, had a better prognosis. In contrast, the prognosis of the remaining $70 \%$ deemed "unfit" for any leukemia-directed therapy, due to poor performance status, comorbid conditions, or various psychosocial considerations, is even more dismal.

\section{Biology of AML in elderly patients}

The results of clinical trials indicate that older patients with AML treated with intensive therapy suffer more complications, have a higher risk of early death, and a lower rate of remission, as well as a shorter progression-free survival and OS. As mentioned above, to a large extent, this is due to the particular biology of the disease in older patients.
Older patients with AML have a higher incidence of antecedent hematologic disorder and a higher incidence of adverse cytogenetics. ${ }^{5,6}$ Many cases of de novo AML in older patients actually resemble secondary AML, in that they are characterized by stem cell phenotypes, multilineage involvement, and overexpression of genes conferring drug resistance. ${ }^{7}$ The multi-drug resistance 1 (MDRI) gene which encodes for P-glycoprotein, a chemotherapy efflux pump, occurs in $71 \%$ of older patients over 55 years of age, compared to only $35 \%$ of younger patients. ${ }^{8}$

A retrospective analysis of 968 AML patients from the Southwest Oncology Group trials was one of the more recent studies that emphasized the low incidence of favorable and 
relatively high incidence of unfavorable cytogenetics in older patients: favorable cytogenetics were found in $17 \%$ versus $4 \%$ and unfavorable cytogenetics in $35 \%$ versus $51 \%$ of patients $<56$ and $>75$ years old, respectively. ${ }^{4}$ The incidence of monosomal karyotype (MK), which carries a particularly dismal prognosis even among "unfavorable karyotypes," was reported at about $20 \%$ in patients $>60$ years old, and only $4 \%$ in patients $<30$ years old. ${ }^{9}$

Cytogenetic features are considered the most independent and strongest predictor of outcome in this disease across all ages. ${ }^{4,10,11}$ A UK Medical Research Council (MRC) AML11 trial of 1,065 patients over 55 years old who were fit to tolerate intensive therapy validated the prognostic significance of cytogenetic groups devised for younger patients in the older patient population. Older patients with favorable karyotype accrued to the AML 11 trial, accounting for $7 \%$ of the participants, enjoyed a CR rate of $72 \%$ and 5 -year OS of $34 \%$. In contrast, older patients with adverse karyotype, accounting for $13 \%$ of all patients in the trial, had a CR of $26 \%$ and a 5 -year OS of $2 \% .{ }^{10}$ This data emphasized the need and provided the framework for stratified treatment approaches in older patient populations with this disease.

However, most elderly individuals have an intermediate cytogenetic risk, which the MRC AML11 trial defined as the presence of unfavorable, non-complex karyotypic abnormalities as well as the presence of normal karyotype. This is different from younger patients, in whom we can clearly appreciate the difference in outcome between patients with normal karyotype and patients with unfavorable non-complex karyotype.

The last few years brought about great progress in the characterization of cytogenetically normal AML. Among those patients, we can now define relatively homogeneous subsets of patients characterized by molecular events such as fms-like tyrosine kinase 3 (FLT3) gene mutation, nucleophosmin 1 (NPM1) gene mutation, CCAAT/enhancer-binding protein alpha (CEBPA) gene mutation, and others. The presence of FLT3 internal tandem duplications (ITDs) in younger patients with AML has been associated with poor prognosis due to increased relapse rate and reduced remission duration and OS. ${ }^{12,13}$ A German study confirmed the poor prognostic bearing of FLT3-ITD mutation in elderly AML with comparable CR but significantly worse OS compared to wild-type patients. ${ }^{14}$ The Cancer and Leukemia Group B (CALGB) study, investigating the significance of FLT3-ITD mutation in older patients with AML, concurred with previous findings of shorter disease-free survival and OS. ${ }^{15}$ Interestingly, the impact of FLT3-ITD mutation was observed particularly in patients aged 60-69 years $(P<0.001)$ and not necessarily in those over 70 years old. ${ }^{15}$
In contrast, the presence of $N P M 1$ mutation in the absence of FLT3 mutation is associated with improved prognosis in younger patients as well as older ones. ${ }^{16}$ The CALGB showed that in elderly patients over 60 years of age, those with NPM1 mutation had a complete remission rate of $84 \%$, as compared to $48 \%$ with wild-type $N P M 1 .{ }^{17}$ The 3 -year OS rates were also higher in those with NPM1 mutations (35\% versus [vs] $8 \%$ ). Similarly, a German study of 99 elderly AML patients demonstrated a higher CR rate of $80 \%$ in NPM1-positive patients as compared to $40.5 \%$ in wild-type NPM1. However, the authors found no significant difference in OS between the two groups. ${ }^{14}$

Interestingly, the German group led by Buchner, in their study of 1,284 AML patients receiving induction chemotherapy, reported a similar incidence of FLT3 and/or NPM1 mutations in patients across all age groups. ${ }^{18}$ As with the overall AML population, there was poorer survival and remission duration in the older versus younger patients regardless of NPM1/FLT3 mutation status. However, older patients with NPM1 mutation performed better than those with wild-type NPM1, and those with FLT3 mutation performed worse than patients without mutated FLT3. ${ }^{18}$

\section{Patient-related factors}

The presence of patient-related factors, such as poor functional status and comorbidities, greatly contribute to poor outcomes of treatment in elderly patients with AML. Due to these factors, older patients are often offered less intensive therapy, which may naturally be less effective. The pharmacokinetic and pharmacodynamic changes that occur with age result in delayed drug clearance, which may add to increased toxicity. ${ }^{6}$ The standard therapeutic strategies in older patients tend to induce deeper and more prolonged myelosuppression, which is likely a major determinant of early treatment-related morbidity and mortality seen in the course of induction in these patients. While older but otherwise fit and healthy patients may be able to tolerate these changes, the toxicity levels in those less fit and less healthy are often prohibitive. The 30-day mortality following aggressive induction chemotherapy for elderly patients with a performance status of $\geq 2$ increases with age and ranges from $18 \%-24 \%$ for patients aged 56-65 years to $50 \%-82 \%$ for patients older than 75 years. ${ }^{4}$ Thus, novel treatment strategies developed specifically for older patients and studied in elderly-specific clinical trials are desperately needed. Such trials should incorporate a comprehensive assessment of older adults in regards to tumor biology as well as comorbidities and functional impairments. Last but not least, we should also start appreciating other 
psychosocial factors such as the availability of caretakers and proximity to trial enrollment centers. ${ }^{19}$

\section{Rationale for treating elderly AML patients}

Given the suboptimal response to treatment and substantial toxicity associated with intensive therapy for elderly AML patients, the potential benefits of such measures have been repeatedly questioned. Using data from the SEER program and Medicare administrative claims, Menzin et al reported that between 1991 and 1996, only 30\% of newly diagnosed AML patients aged 65 years and older underwent chemotherapy. ${ }^{20}$ The median OS for all patients was only 2 months and 2-year survival was only $6 \%$. However, the median survival of patients who received chemotherapy was 8 months. ${ }^{20}$ The follow-up SEER-Medicare data from 1999-2002 found that administration of chemotherapy to elderly AML patients increased to $36 \% .^{21}$ As expected, the median survival in the treated group was longer than the untreated group (6.1 months vs 1.7 months). Obviously, such data is difficult to interpret, as patients who were offered treatment were likely younger and more fit than those who did not.

However, newer data generated by randomized trials and new, larger database analyses definitely indicate that elderly patients with AML treated with aggressive therapy can achieve durable remissions, and that this translates into prolongation of survival and good quality of life. ${ }^{22-24}$

The landmark publication by Lowenberg et al compared immediate-intensive chemotherapy versus supportive care with mild cytoreductive chemotherapy for AML-related symptom relief in patients older than 65 years of age. ${ }^{22}$ Intensive chemotherapy with daunorubicin, vincristine, and cytarabine was associated with high CR rates (58\% vs $0 \%)$, longer median survival (21 weeks vs 11 weeks), and improved disease-free survival at 2.5 years $(13 \%$ vs $0 \%)$. More importantly, the authors showed that there was no difference in the percentage of days the patient spent in the hospital. Population-based data from the Swedish Acute Leukemia Registry strongly suggests that standard intensive chemotherapy improves early mortality and long-term survival compared with palliation. ${ }^{23}$ A more recent publication from Japan also found intensive chemotherapy in elderly AML was beneficial. ${ }^{24}$

Although better than supportive care, the results of intensive therapy in elderly patients remain disappointing. Numerous clinical trials have looked into increasing or attenuating doses, adding or replacing drugs, and utilizing growth factors - without much success. Lowenberg et al conducted a
Phase III study comparing the standard " $7+3$ regimen" with standard-dose $\left(45 \mathrm{mg} / \mathrm{m}^{2}\right)$ versus intensified-dose $\left(90 \mathrm{mg} / \mathrm{m}^{2}\right)$ of daunorubicin in AML patients aged 60 years or older. ${ }^{25}$ Although there was no overall difference in outcome between the two groups, the intensified-dose was associated with increased response rates and survival benefit in a subset of patients aged 60-65 years. Schlenk et al evaluated the impact of all-trans retinoic acid (ATRA) as an adjunct to induction and consolidation chemotherapy in AML patients 60 years or older. ${ }^{26}$ The addition of ATRA during induction chemotherapy significantly increased CR (52\% vs 39\%), event-free survival (EFS), and OS rates. More recently, a French study evaluated the effect of adding gemtuzumab ozogamicin (GO), an anti-CD33 antibody conjugate, to standard chemotherapy regimens in newly diagnosed AML patients aged 50-70 years. ${ }^{27}$ Although the CR rates were similar in both groups, the addition of $\mathrm{GO}$ significantly improved the 2-year EFS (40.8\% vs $17.1 \%$ ) and OS (53.2\% vs 21.9\%) rates. Similarly, in the UKAML 16 study, the addition of GO on day 1 of induction chemotherapy showed a small but significant 3-year OS benefit (25\% vs $20 \%$ ) with no increase in toxicity. ${ }^{28}$ Both these studies have generated renewed interest in gemtuzumab, which was voluntarily withdrawn by Pfizer from the US market in 2010 due to lack of efficacy in the presence of enhanced toxicity. ${ }^{29}$ Regardless, aggressive therapy for older patients with AML remains only modestly effective overall. ${ }^{30}$

Perhaps the greatest achievement of the past decade in this field was a realization that patients unfit to receive aggressive therapy can still benefit from leukemia-specific chemotherapy, albeit at lower doses, which still offers survival advantage and improved quality of life.

An MRC AML 14 trial randomly selected 217 elderly AML patients deemed unsuitable for intensive chemotherapy to receive low-dose cytarabine or hydroxyurea with best supportive care. ${ }^{31}$ Low-dose cytarabine was associated with significantly higher CR rates (18\% vs $1 \%$ ) and 1 -year OS (25\% vs 6\%). Remarkably, the toxicity scores and requirement for supportive care did not differ between trial arms. Additionally, the benefit in this trial was limited to patients with favorable and intermediate risk cytogenetics, and the overall prognosis remained quite poor with the OS for the entire study population being $13 \%$ at 1 year, $4 \%$ at 2 years, and $<1 \%$ at 3 years.

Numerous efforts have been undertaken to create treatment options that would be tolerable but also more effective. Clofarabine seemed to have quite a favorable toxicity profile and has been tested extensively in American and European 
trials. $^{32,33}$ The trials yielded encouraging results, particularly in older patients with AML associated with poor risk karyotypes. However, despite a few unique toxicities, the safety profile proved very comparable with standard intensive chemotherapy regimens. Clofarabine is currently being compared with the standard of care combination of anthracycline and cytarabine in an Eastern Cooperative Oncology Group (ECOG)-led Intergroup Phase III Randomized Trial. ${ }^{34}$

Demethylating agents offer a novel mechanism of action, acceptable toxicity profile, and proven efficacy in myelodysplastic syndromes, which makes them great candidates for treatment in this setting. A subset analysis of a large Phase III randomized trial compared the effects of azacitidine in elderly patients with low bone marrow blast counts $(20 \%-30 \%)$ with conventional care regimens (CCR), which included intensive chemotherapy (IC), low-dose cytarabine (LDAC), or best supportive care (BSC). ${ }^{35}$ Of the 113 elderly patients, with a median age of 70 years, $86 \%$ were considered unfit for IC. In the CCR group, $47 \%$ received BSC, $34 \%$ received LDAC, and only $19 \%$ received IC. The authors found that azacitidine significantly prolonged OS (24.5 months vs 16.0 months). ${ }^{35}$ Notably the 2-year survival in patients with adverse cytogenetics was strongly in favor of azacitidine (38\% vs $0 \%)$. Decitabine has also been evaluated in elderly AML patients unsuitable for intensive chemotherapy. ${ }^{36-38}$ In a multicenter Phase III trial, 485 elderly AML patients aged $\geq 65$ years were randomly assigned to receive either decitabine $20 \mathrm{mg} / \mathrm{m}^{2}$ per day as a 1-hour intravenous infusion for 5 consecutive days every 4 weeks or treatment choice (TC) (supportive care or cytarabine $20 \mathrm{mg}$ / $\mathrm{m}^{2}$ per day as a subcutaneous injection for 10 consecutive days every 4 weeks). ${ }^{38}$ Treatment with decitabine was associated with a higher CR rate and better median OS (7.7 months vs 5.0 months). Although the difference in survival was not statistically significant, and thus, the study failed to meet its primary objective, this data eventually led to the approval of decitabine for the treatment of AML in adults 65 years or older by the European Commission. The drug was not approved for the treatment of AML in the US although further studies of decitabine in the US resulted in the creation of a prolonged 10-day dosing regimen, which may prove more effective than the original 5-day regimen adapted from myelodysplastic syndrome (MDS). ${ }^{37}$ The 10-day regimen is currently being investigated by a CALGB-led intergroup randomized trial. ${ }^{39}$

A few other agents are in the advanced stages of development, including but not limited to CPX-351, which fixes a 5:1 ratio of cytarabine to daunorubicin within a liposomal carrier and a novel topoisomerase II inhibitor, vosaroxin. ${ }^{40,41}$ Hopefully, the next few years will see significant progress in the development of more effective and less toxic regimens. However, it is not apparent to what extent these treatment modalities will be available to help older patients with AML who are unable to travel to the chemotherapy treatment centers daily to receive their decitabine or every other day to check their blood counts and receive their blood products. In this regard, sapacitabine, a novel oral nucleoside analog holds great promise as it can be given orally at home and may not require intensive supportive care.

\section{Profile of sapacitabine}

Sapacitabine (CS-682) is an oral palmitoyl derivative of 2 '-C-Cyano- 2 '-deoxy- $\beta$-D-arabino-pentofuranosylcytosine (CNDAC), a deoxycytidine analog with a novel DNA-strandbreaking mechanism of action (Figure 5). ${ }^{42,43}$ The fatty-acid side chain on the N4 group of the cytosine moiety improves the oral bioavailability of sapacitabine and reduces inactivation by deamination. Upon oral administration, sapacitabine is converted to its active metabolite, CNDAC, by amidases and esterases in the plasma, gut, and liver. CNDAC is then phosphorylated to CNDAC-triphosphate by intracellular deoxycytidine kinase before being incorporated into cellular DNA. Following its integration into DNA, CNDAC inhibits DNA polymerases, resulting in cessation of DNA-strand elongation. In addition, CNDAC promotes induction of single-strand DNA breaks, which are converted to doublestrand DNA breaks, thus activating the double-stranded DNA (dsDNA) repair pathway and resulting in arrest at the G2/M phase. This mechanism is unique and not seen in other deoxycytidine analogs such as cytarabine or gemcitabine..$^{44,45}$

\section{Preclinical studies}

Preclinical studies have demonstrated the potential of sapacitabine and CNDAC for antitumor activity. Sapacitabine displays cytotoxic activity across a range of concentrations in a variety of human solid tumor cell lines. ${ }^{46} \mathrm{CNDAC}$ was found to be more effective than cytarabine in murine and human tumor cell lines derived from lung, stomach, and osteosarcoma samples. ${ }^{47}$ However, sapacitabine appeared to be more potent than CNDAC against human organ tumor xenografts at a wide dose range and with low toxicity. ${ }^{43}$ Sapacitabine was also effective against P388 human leukemia cells resistant to mitomycin-C, vincristine, 5-fluorouracil, or cisplatin in syngeneic mice. ${ }^{43}$ In an orthotopic human pancreatic cancer mouse model, sapacitabine suppressed the rate of primary tumor growth, decreased the formation of metastases, and significantly prolonged survival at all doses compared with untreated animals. ${ }^{48,49}$ The combination of sapacitabine and histone deacetylase (HDAC) inhibitors induced a significant 

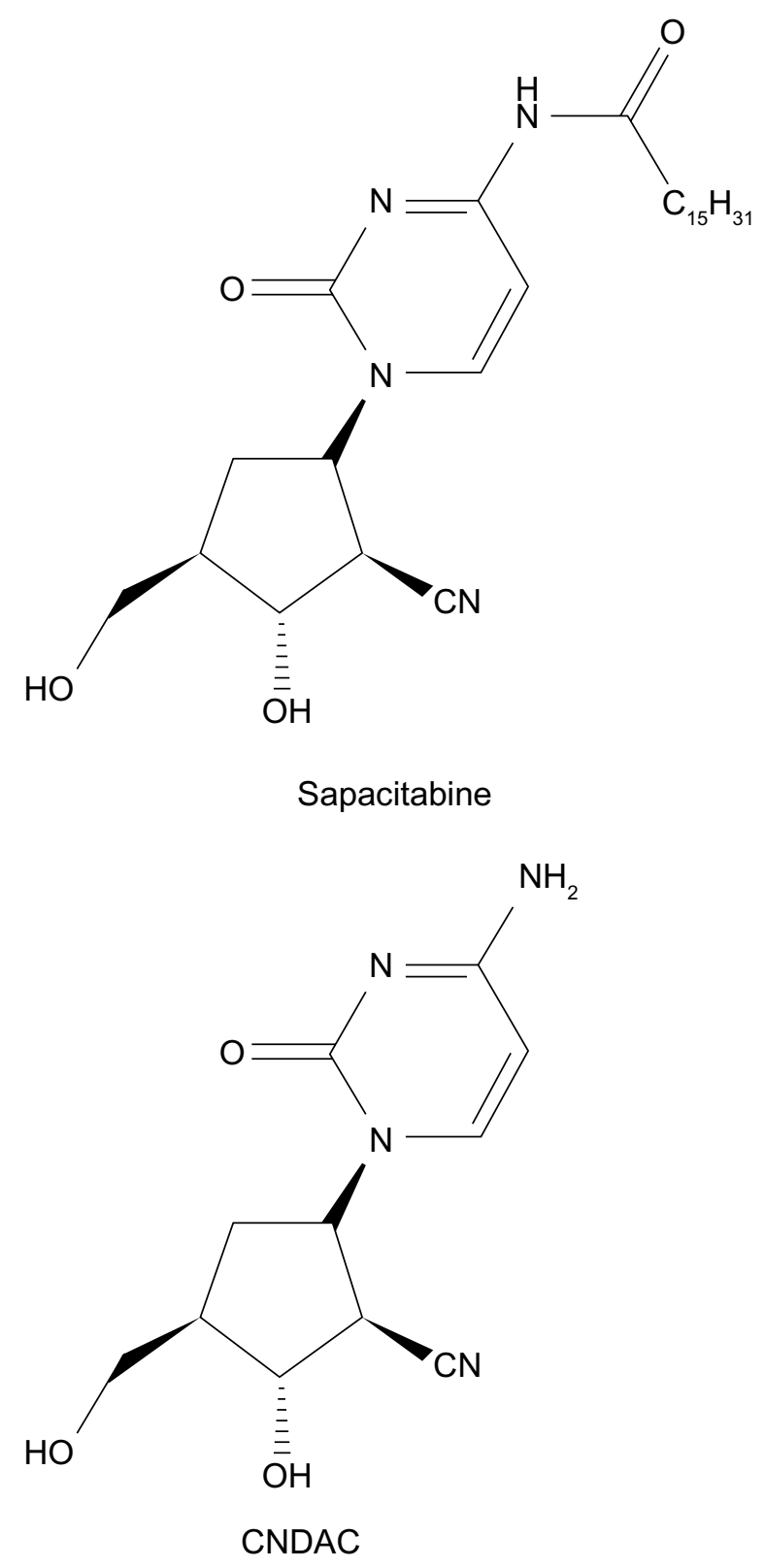

Figure 5 Structure of sapacitabine and CNDAC.

Abbreviation: CNDAC, 2'-C-Cyano-2'-deoxy- $\beta$-D-arabino-pentofuranosylcytosine.

increase in apoptosis in an AML cell line, MV4-11, in vitro and in an MV4-11 mouse xenograft model in vivo. ${ }^{50}$

\section{Clinical trials}

Encouraging results from preclinical studies have led to several Phase I studies of sapacitabine in solid tumors. Sapacitabine was administered orally to 48 patients with refractory solid tumor malignancies. ${ }^{51}$ No objective tumor response was noted, although eleven patients achieved disease stability. More importantly, the drug was very welltolerated and had very few side effects, with the dose-limiting toxicity being neutropenia. A second study of 40 patients with advanced solid tumors also did not demonstrate any tumor response, although six patients experienced disease stability. ${ }^{52}$ Because of its unique mechanism of action, and a defined dose-limiting toxicity of neutropenia in solid tumors, sapacitabine became an attractive agent to investigate in hematological malignancies.

Kantarjian et al conducted a Phase I trial of sapacitabine in 47 patients aged 18 years or older with refractory-relapsed acute leukemia, myelodysplastic syndrome, or untreated disease, who received sapacitabine in escalating doses from $75 \mathrm{mg}$ to $375 \mathrm{mg}$ twice daily for 7 days $(\mathrm{n}=35)$ and from $375 \mathrm{mg}$ to $475 \mathrm{mg}$ twice daily for 3 days per week for 2 weeks $(n=12) .{ }^{53}$ In both dosing regimens, the dose-limiting non-hematological toxicity was gastrointestinal symptoms of diarrhea and neutropenic colitis. The overall response rate was $28 \%(n=13)$, with four patients $(9 \%)$ achieving complete remission. The estimated 4 -week mortality was only $4 \%$. This study established a maximum tolerated dose of $325 \mathrm{mg}$ twice daily for 7 days or $425 \mathrm{mg}$ twice daily on days 1 to 3 weekly for 2 weeks.

The encouraging anti-leukemic activity of sapacitabine, its reasonable safety profile, and more importantly, its ease of oral administration, makes sapacitabine an ideal drug for study in the elderly AML population. A multi-institutional randomized Phase II study recruited 105 AML patients $>70$ years who were either treatment-naïve $(n=86)$ or at first relapse $(n=19) .{ }^{54}$ The patients were randomized to a three-arm trial of sapacitabine at $200 \mathrm{mg}$ twice daily for 7 days every 28 days (Arm A), at $300 \mathrm{mg}$ on the same schedule (Arm B), or at $400 \mathrm{mg}$ twice daily for 3 days per week for 2 weeks, every 28 days (Arm C). The overall 1-year survival was 35\%,10\%, and 30\% and median OS was 197, 102, and 213 days in Arms A, B, and C, respectively. Although sapacitabine was well-tolerated with a 30 -day and 60-day mortality of $13 \%$ and $26 \%$, respectively, frequent dose reduction was required due to myelosuppression, which could potentially affect its efficacy.

The encouraging results of sapacitabine have led to further trials evaluating sapacitabine in combination with other currently available agents, such as decitabine. In a pilot Phase I/II study, 46 elderly AML patients were treated with alternating cycles of decitabine $20 \mathrm{mg} / \mathrm{m}^{2}$ intravenously daily $\times 5$ days of a 4-week cycle (odd cycles) and sapacitabine $300 \mathrm{mg}$ orally twice daily $\times 3$ days/week of a 4 -week cycle (even cycles). ${ }^{55}$ The median age was 77 years (range 70-90 years) and the median OS was 238 days, approximately 8 months. The 3-, 6-, 12-, and 18-month survival rates were $83 \%, 65 \%, 35 \%$, and $26 \%$, respectively. Nineteen patients (41\%) responded with ten CRs, four partial responses (PRs), and five major hematological improvements (HIs). The median response 
time was two cycles, ie, one cycle of decitabine and one cycle of sapacitabine (range 1-10). Thirty-day mortality from all causes was $4 \%$.

\section{Ongoing clinical trials}

The safety and efficacy of the sequential combination of decitabine and sapacitabine has led to the creation of a Study of Oral Sapacitabine in Elderly Patients with Newly Diagnosed Acute Myeloid Leukemia (SEAMLESS); a pivotal, multicenter, randomized Phase III study comparing sapacitabine and/or decitabine as front-line treatment of elderly patients aged 70 years or older with newly diagnosed AML who are not candidates for intensive induction chemotherapy. ${ }^{56}$ Patients receive either sapacitabine administered in alternating cycles with decitabine, or decitabine alone. The primary efficacy endpoint is OS. The study is expected to complete accrual in April 2015.

\section{Conclusion}

Decisions regarding the optimal treatment in individual older patients with AML require consideration of multiple factors and remain quite challenging. As reviewed above, numerous studies published in recent years consistently demonstrated that a specific anti-leukemia therapy results in improved survival and quality of life when compared with best supportive care. We no longer need "to protect" elderly patients from therapy. However, improvements observed with currently available treatment approaches are modest at best and the overall prognosis remains poor.

This invites renewed efforts to investigate anti-leukemic therapies specifically designed to treat elderly patients with AML; therapies that will take into account the different biology of the disease as well as offer favorable toxicity profiles and relative ease of administration. The future of leukemia treatment clearly lies in targeted therapy. Many of the newer targeted drugs are better tolerated than conventional chemotherapy and should be well-tolerated by younger as well as older patients. At this time, however, having drugs that could be taken at home and that could provide improvement in survival and quality of life would be greatly appreciated not only by patients but also by their physicians. The SEAMLESS trial fulfills a great need as it strives to provide access to potentially effective therapy to patients over 70 years old. These patients, due to their age, comorbid conditions, and/or inadequate social support, are offered treatment only occasionally. Home therapy should at least partly alleviate the need for caregivers and transportation. With all this in mind, little is known about the true impact of such treatment delivery on the patient and/or family. It will be very interesting to learn if such treatment will truly improve the lives of patients and their families. The medical community is awaiting the results from this trial with great interest.

\section{Disclosure}

The authors report no conflicts of interest in this work.

\section{References}

1. Howlader N, Noone AM, Krapcho M, et al (editors). SEER Cancer Statistics Review, 1975-2010. Bethesda, MD: National Cancer Institute; 2013.

2. Burnett AK, Mohite U. Treatment of older patients with acute myeloid leukemia - new agents. Semin Hematol. 2006;43(2):96-106.

3. Derolf AR, Kristinsson SY, Andersson TM, Landgren O, Dickman PW, Bjorkholm M. Improved patient survival for acute myeloid leukemia: a population-based study of 9729 patients diagnosed in Sweden between 1973 and 2005. Blood. 2009;113(16):3666-3672.

4. Appelbaum FR, Gundacker H, Head DR, et al. Age and acute myeloid leukemia. Blood. 2006;107(9):3481-3485.

5. Hoyle CF, de Bastos M, Wheatley K, et al. AML associated with previous cytotoxic therapy, MDS or myeloproliferative disorders: results from the MRC's 9th AML trial. Br J Haematol. 1989;72(1):45-53.

6. Pollyea DA, Kohrt HE, Medeiros BC. Acute myeloid leukaemia in the elderly: a review. Br J Haematol. 2011;152(5):524-542.

7. Head DR. Revised classification of acute myeloid leukemia. Leukemia. 1996;10(11):1826-1831.

8. Leith CP, Kopecky KJ, Godwin J, et al. Acute myeloid leukemia in the elderly: assessment of multidrug resistance (MDR1) and cytogenetics distinguishes biologic subgroups with remarkably distinct responses to standard chemotherapy. A Southwest Oncology Group study. Blood. 1997;89(9):3323-3329.

9. Medeiros BC, Othus M, Fang M, Roulston D, Appelbaum FR. Prognostic impact of monosomal karyotype in young adult and elderly acute myeloid leukemia: the Southwest Oncology Group (SWOG) experience. Blood. 2010;116(13):2224-2228.

10. Grimwade D, Walker H, Harrison G, et al. The predictive value of hierarchical cytogenetic classification in older adults with acute myeloid leukemia (AML): analysis of 1065 patients entered into the United Kingdom Medical Research Council AML11 trial. Blood. 2001;98(5):1312-1320.

11. Farag SS, Archer KJ, Mrozek K, et al. Pretreatment cytogenetics add to other prognostic factors predicting complete remission and longterm outcome in patients 60 years of age or older with acute myeloid leukemia: results from Cancer and Leukemia Group B 8461. Blood. 2006;108(1):63-73.

12. Frohling S, Schlenk RF, Breitruck J, et al. Prognostic significance of activating FLT3 mutations in younger adults (16 to 60 years) with acute myeloid leukemia and normal cytogenetics: a study of the AML Study Group Ulm. Blood. 2002;100(13):4372-4380.

13. Yanada M, Matsuo K, Suzuki T, Kiyoi H, Naoe T. Prognostic significance of FLT3 internal tandem duplication and tyrosine kinase domain mutations for acute myeloid leukemia: a meta-analysis. Leukemia. 2005;19(8):1345-1349.

14. Scholl S, Theuer C, Scheble V, et al. Clinical impact of nucleophosmin mutations and Flt3 internal tandem duplications in patients older than $60 \mathrm{yr}$ with acute myeloid leukaemia. Eur J Haematol. 2008;80(3):208-215.

15. Whitman SP, Maharry K, Radmacher MD, et al. FLT3 internal tandem duplication associates with adverse outcome and gene- and microRNAexpression signatures in patients 60 years of age or older with primary cytogenetically normal acute myeloid leukemia: a Cancer and Leukemia Group B study. Blood. 2010;116(18):3622-3626. 
16. Dohner K, Schlenk RF, Habdank M, et al. Mutant nucleophosmin (NPM1) predicts favorable prognosis in younger adults with acute myeloid leukemia and normal cytogenetics: interaction with other gene mutations. Blood. 2005;106(12):3740-3746.

17. Becker H, Marcucci G, Maharry K, et al. Favorable prognostic impact of NPM1 mutations in older patients with cytogenetically normal de novo acute myeloid leukemia and associated gene- and microRNAexpression signatures: a Cancer and Leukemia Group B study. J Clin Oncol. 2010;28(4):596-604.

18. Buchner T, Berdel WE, Haferlach C, et al. Age-related risk profile and chemotherapy dose response in acute myeloid leukemia: a study by the German Acute Myeloid Leukemia Cooperative Group. J Clin Oncol. 2009;27(1):61-69.

19. Gross CP, Herrin J, Wong N, Krumholz HM. Enrolling older persons in cancer trials: the effect of sociodemographic, protocol, and recruitment center characteristics. J Clin Oncol. 2005;23(21):4755-4763.

20. Menzin J, Lang K, Earle CC, Kerney D, Mallick R. The outcomes and costs of acute myeloid leukemia among the elderly. Arch Intern Med. 2002;162(14):1597-1603.

21. Menzin J, Boulanger L, Karsten V, Cahill AL, Earle CE. Effects of initial treatment on survival among elderly AML patients: findings from the SEER-Medicare Database. ASH Annual Meeting Abstracts. 2006;108:1973.

22. Lowenberg B, Zittoun R, Kerkhofs H, et al. On the value of intensive remission-induction chemotherapy in elderly patients of $65+$ years with acute myeloid leukemia: a randomized phase III study of the European Organization for Research and Treatment of Cancer Leukemia Group. J Clin Oncol. 1989;7(9):1268-1274.

23. Juliusson G, Antunovic P, Derolf A, et al. Age and acute myeloid leukemia: real world data on decision to treat and outcomes from the Swedish Acute Leukemia Registry. Blood. 2009;113(18):4179-4187.

24. Oshima K, Takahashi W, Asano-Mori Y, et al. Intensive chemotherapy for elderly patients with acute myelogeneous leukemia: a propensity score analysis by the Japan Hematology and Oncology Clinical Study Group (J-HOCS). Ann Hematol. 2012;91(10):1533-1539.

25. Lowenberg B, Ossenkoppele GJ, van Putten W, et al. High-dose daunorubicin in older patients with acute myeloid leukemia. $N$ Engl J Med. 2009;361(13):1235-1248.

26. Schlenk RF, Frohling S, Hartmann F, et al. Phase III study of all-trans retinoic acid in previously untreated patients 61 years or older with acute myeloid leukemia. Leukemia. 2004;18(11):1798-1803.

27. Castaigne S, Pautas C, Terre C, et al. Effect of gemtuzumab ozogamicin on survival of adult patients with de-novo acute myeloid leukaemia (ALFA-0701): a randomised, open-label, phase 3 study. Lancet. 2012;379(9825):1508-1516.

28. Burnett AK, Russell NH, Hills RK, et al. Addition of gemtuzumab ozogamicin to induction chemotherapy improves survival in older patients with acute myeloid leukemia. J Clin Oncol. 2012;30(32):3924-3931.

29. US Food and Drug Administration [webpage on the Internet] Gemtuzumab ozogamicin [last updated June 22, 2010]. Available from: http://www.fda.gov/AboutFDA/CentersOffices/OfficeofMedicalProdu ctsandTobacco/CDER/ucm216790.htm. Accessed March 11, 2014.

30. Klepin HD, Balducci L. Acute myelogenous leukemia in older adults. Oncologist. 2009;14(3):222-232.

31. Burnett AK, Milligan D, Prentice AG, et al. A comparison of low-dose cytarabine and hydroxyurea with or without all-trans retinoic acid for acute myeloid leukemia and high-risk myelodysplastic syndrome in patients not considered fit for intensive treatment. Cancer. 2007;109(6): 1114-1124.

32. Burnett AK, Russell NH, Kell J, et al. European development of clofarabine as treatment for older patients with acute myeloid leukemia considered unsuitable for intensive chemotherapy. J Clin Oncol. 2010;28(14):2389-2395.

33. Kantarjian HM, Erba HP, Claxton D, et al. Phase II study of clofarabine monotherapy in previously untreated older adults with acute myeloid leukemia and unfavorable prognostic factors. J Clin Oncol. 2010;28(4): 549-555.
34. ClinicalTrials/gov. Eastern Cooperative Oncology Group. Clofarabine or Daunorubicin Hydrochloride and Cytarabine Followed By Decitabine or Observation in Treating Older Patients With Newly Diagnosed Acute Myeloid Leukemia. Available from: http:/clinicaltrials.gov/show/ NCT01041703. NLM identifier: NCT01041703. Accessed April 3, 2014.

35. Fenaux P, Mufti GJ, Hellstrom-Lindberg E, et al. Azacitidine prolongs overall survival compared with conventional care regimens in elderly patients with low bone marrow blast count acute myeloid leukemia. J Clin Oncol. 2010;28(4):562-569.

36. Cashen AF, Schiller GJ, O’Donnell MR, DiPersio JF. Multicenter, phase II study of decitabine for the first-line treatment of older patients with acute myeloid leukemia. J Clin Oncol. 2010;28(4): $556-561$.

37. Blum W, Garzon R, Klisovic RB, et al. Clinical response and miR-29b predictive significance in older AML patients treated with a 10-day schedule of decitabine. Proc Nat Acad Sci U S A. 2010;107(16): 7473-7478

38. Kantarjian HM, Thomas XG, Dmoszynska A, et al. Multicenter, randomized, open-label, phase III trial of decitabine versus patient choice, with physician advice, of either supportive care or low-dose cytarabine for the treatment of older patients with newly diagnosed acute myeloid leukemia. J Clin Oncol. 2012;30(21):2670-2677.

39. ClinicalTrials.gov. National Cancer Institute (NCI). Decitabine With or Without Bortezomib in Treating Older Patients With Acute Myeloid Leukemia. Available from: http://clinicaltrials.gov/show/NCT01420926. NLM Identifier: NCT01420926. Accessed April 3,2014

40. Feldman EJ, Kolitz JE, Trang JM, et al. Pharmacokinetics of CPX-351; a nano-scale liposomal fixed molar ratio formulation of cytarabine:daunorubicin, in patients with advanced leukemia. Leuk Res. 2012;36(10):1283-1289.

41. Freeman C, Keane N, Swords R, Giles F. Vosaroxin: a new valuable tool with the potential to replace anthracyclines in the treatment of AML? Expert Opin Pharmacother. 2013;14(10):1417-1427.

42. Matsuda A, Nakajima Y, Azuma A, Tanaka M, Sasaki T. Nucleosides and nucleotides. 100. 2'-C-cyano-2'-deoxy-1-beta-D-arabinofuranosylcytosine (CNDAC): design of a potential mechanism-based DNAstrand-breaking antineoplastic nucleoside. J Med Chem. 1991;34(9): 2917-2919.

43. Hanaoka K, Suzuki M, Kobayashi T, et al. Antitumor activity and novel DNA-self-strand-breaking mechanism of CNDAC (1-(2-C-cyano-2deoxy-beta-D-arabino-pentofuranosyl) cytosine) and its N4-palmitoyl derivative (CS-682). Int J Cancer. 1999;82(2):226-236.

44. Azuma A, Huang P, Matsuda A, Plunkett W. 2'-C-cyano-2'-deoxy-1beta-D-arabino-pentofuranosylcytosine: a novel anticancer nucleoside analog that causes both DNA strand breaks and G(2) arrest. Mol Pharmacol. 2001;59(4):725-731.

45. Liu X, Matsuda A, Plunkett W. Ataxia-telangiectasia and Rad3-related and DNA-dependent protein kinase cooperate in G2 checkpoint activation by the DNA strand-breaking nucleoside analogue 2 '-Ccyano-2'-deoxy-1-beta-D-arabino-pentofuranosylcytosine. Mol Cancer Ther. 2008;7(1):133-142.

46. Serova M, Galmarini CM, Ghoul A, et al. Antiproliferative effects of sapacitabine (CYC682), a novel 2'-deoxycytidine-derivative, in human cancer cells. Br J Cancer. 2007;97(5):628-636.

47. Tanaka M, Matsuda A, Terao T, Sasaki T. Antitumor activity of a novel nucleoside, 2'-C-cyano-2'-deoxy-1-beta-D-arabinofuranosylcytosine (CNDAC) against murine and human tumors. Cancer Lett. 1992;64(1): 67-74.

48. Katz MH, Bouvet M, Takimoto S, Spivack D, Moossa AR, Hoffman RM. Survival efficacy of adjuvant cytosine-analogue CS-682 in a fluorescent orthotopic model of human pancreatic cancer. Cancer Res. 2004;64(5):1828-1833.

49. Katz MH, Bouvet M, Takimoto S, Spivack D, Moossa AR, Hoffman RM. Selective antimetastatic activity of cytosine analog CS-682 in a red fluorescent protein orthotopic model of pancreatic cancer. Cancer Res. 2003;63(17):5521-5525. 
50. Green SR, Choudhary AK, Fleming IN. Combination of sapacitabine and HDAC inhibitors stimulates cell death in AML and other tumour types. Br J Cancer. 2010;103(9):1391-1399.

51. Gilbert J, Carducci MA, Baker SD, Dees EC, Donehower R. A Phase I study of the oral antimetabolite, CS-682, administered once daily 5 days per week in patients with refractory solid tumor malignancies. Invest New Drugs. 2006;24(6):499-508.

52. Delaunoit T, Burch PA, Reid JM, et al. A phase I clinical and pharmacokinetic study of CS-682 administered orally in advanced malignant solid tumors. Invest New Drugs. 2006;24(4):327-333.

53. Kantarjian H, Garcia-Manero G, O'Brien S, et al. Phase I clinical and pharmacokinetic study of oral sapacitabine in patients with acute leukemia and myelodysplastic syndrome. J Clin Oncol. 2010;28(2): 285-291.
54. Kantarjian H, Faderl S, Garcia-Manero G, et al. Oral sapacitabine for the treatment of acute myeloid leukaemia in elderly patients: a randomised phase 2 study. Lancet Oncol. 2012;13(11):1096-1104.

55. Ravandi F, Kadia TM, Borthakur G, et al. Pooled analysis of elderly patients with newly diagnosed AML treated with sapacitabine and decitabine administered in alternating cycles. Poster presented at: Blood (ASH Annual Meeting Abstracts); December 9, 2012; Atlanta, Georgia: Abst 2630.

56. ClinicalTrials.gov. Cyclacel Pharmaceuticals, Inc. A Study of Oral Sapacitabine in Elderly Patients With Newly Diagnosed Acute Myeloid Leukemia (SEAMLESS). Available from: http://clinicaltrials.gov/show/ NCT01303796. NLM Identifier: NCT01303796. Accessed April 3, 2014.
Clinical Interventions in Aging

\section{Publish your work in this journal}

Clinical Interventions in Aging is an international, peer-reviewed journal focusing on evidence-based reports on the value or lack thereof of treatments intended to prevent or delay the onset of maladaptive correlates of aging in human beings. This journal is indexed on PubMed Central, MedLine, the American Chemical Society's 'Chemical Abstracts Ser-

\section{Dovepress}

vice' (CAS), Scopus and the Elsevier Bibliographic databases. The manuscript management system is completely online and includes a very quick and fair peer-review system, which is all easy to use. Visit $\mathrm{http} / / / \mathrm{www}$.dovepress.com/testimonials.php to read real quotes from published authors.

Submit your manuscript here: http://www.dovepress.com/clinical-interventions-in-aging-journal 